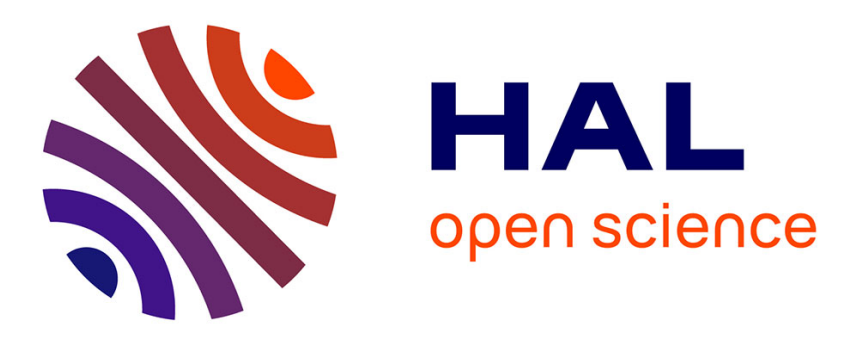

\title{
Possible planet formation in the young, low-mass, multiple stellar system GG Tau A
}

Anne Dutrey, E. Di Folco, S. Guilloteau, Y. Boehler, Jeff Bary, Tracy Beck, Hervé Beust, E. Chapillon, Fredéric Gueth, J.-M. Huré, et al.

\section{- To cite this version:}

Anne Dutrey, E. Di Folco, S. Guilloteau, Y. Boehler, Jeff Bary, et al.. Possible planet formation in the young, low-mass, multiple stellar system GG Tau A. Nature, 2014, 514 (7524), pp.600-602. 10.1038/nature13822. hal-01078717v2

\section{HAL Id: hal-01078717 https://hal.science/hal-01078717v2}

Submitted on 4 Nov 2014

HAL is a multi-disciplinary open access archive for the deposit and dissemination of scientific research documents, whether they are published or not. The documents may come from teaching and research institutions in France or abroad, or from public or private research centers.
L'archive ouverte pluridisciplinaire HAL, est destinée au dépôt et à la diffusion de documents scientifiques de niveau recherche, publiés ou non, émanant des établissements d'enseignement et de recherche français ou étrangers, des laboratoires publics ou privés. 


\section{Possible Planet formation in the young, low-mass, multi- ple stellar system GGTau-A}

Anne Dutrey $^{1,2}$, Emmanuel Di Folco ${ }^{1,2}$, Stéphane Guilloteau ${ }^{1,2}$, Yann Boehler ${ }^{3}$, Jeff Bary ${ }^{4}$, Tracy Beck $^{5}$, Hervé Beust ${ }^{6}$, Edwige Chapillon ${ }^{1,7}$, Fredéric Gueth ${ }^{7}$, Jean-Marc Huré ${ }^{1,2}$, Arnaud Pierens ${ }^{1,2}$, Vincent Piétu, ${ }^{7}$, Michal Simon ${ }^{8} \&$ Ya-Wen Tang ${ }^{9}$

${ }^{1}$ Univ. Bordeaux, LAB, UMR 5804, F-33270, Floirac, France

${ }^{2}$ CNRS, LAB, UMR 5804, F-33270 Floirac, France

${ }^{3}$ CRyA, University of Mexico, Apartado Postal 3-72, 58089 Morelia, Michoacan, Mexico

${ }^{4}$ Department of Physics and Astronomy, Colgate University, 13 Oak Drive, Hamilton, NY 13346, USA

${ }^{5}$ Space Telescope Science Institute,3700 san Martin Dr. Baltimore, MD 21218, USA

${ }^{6}$ IPAG, UMR 5274, BP 53, F-38041 Grenoble Cedex 9, France

${ }^{7}$ IRAM, 300 rue de la Piscine, F-38046 Saint Martin d'Hères, France

${ }^{8}$ Stony Brook University, Stony Brook, NY 11794-3800, USA

${ }^{9}$ Academia Sinica Institute of Astronomy and Astrophysics, P.O. Box 23-141, Taipei, 106 Taiwan

Forming planets around binary stars may be more difficult than around single $\operatorname{stars}^{1-3}$. In a close binary star ( $<100$ au separation), theory predicts the presence of circumstellar discs around each star, and an outer circumbinary disc surrounding a gravitationally cleared inner cavity ${ }^{4,5}$. As the inner discs are depleted by accretion onto the stars on timescales of few $10^{3} \mathrm{yr}$, replenishing material must be transferred from the outer reservoir in order to fuel 
planet formation (which occurs on timescales of $\sim 1$ Myr). Gas flowing through disc cavities has been detected in single star systems ${ }^{6}$. A circumbinary disc was discovered around the young low-mass binary system GGTau- $\mathrm{A}^{7}$, which has recently been proven to be a hierarchical triple system ${ }^{8}$. It has one large inner $\operatorname{disc}^{9}$ around the southern single star and shows small amounts of shocked $\mathrm{H}_{2}$ gas residing within the central cavity ${ }^{10}$, but other than a weak detection $^{11}$, hitherto the distribution of cold gas in this cavity or in any other binary or multiple star system has never been determined. Here we report imaging of massive CO-emitting gas fragments within the GG Tau-A cavity. From the kinematics we conclude that the flow appears capable of sustaining the inner disc beyond the accretion lifetime, leaving time for planet formation to occur.

The 1-5 Million year old ${ }^{12,13}$ triple stellar system GGTau-A is located at $140 \mathrm{pc}$ in a hole of the Taurus molecular cloud. Its molecular emission is free of contamination ${ }^{14}$ and there is no known outflow neither jet associated to the source. The main binary GGTau-A (Aa-Ab) and the close-binary GGTau-Ab (Ab1-Ab2) have an apparent separation of 35 au and 4.5 au, respectively . $^{8}$ The outer circumbinary Keplerian disc of gas and dust surrounding GGTau-A consists of a ring extending from radius $r \sim 190$ to 280 au and an outer disc extending up to 800 au from the central stars with a total mass $\sim 0.15 \mathrm{M}_{\odot}{ }^{14}$.

Using the Atacama Large Millimetre Array (ALMA), we observed GGTau-A in the dust thermal emission at $0.45 \mathrm{~mm}$ and in $\mathrm{CO} \mathrm{J}=6-5$ line (Fig.1: panels a,b,c) with an angular resolution $\theta \simeq 0.25^{\prime \prime}$ or $\sim 35$ au. The continuum image shows cold dust emission from only one circumstellar 
disc-like structure associated with GGTau-Aa ${ }^{9,15}$. We estimate the minimum dust disc size to be $\sim 7$ au while the minimum mass of gas and dust is roughly $10^{-3} \mathrm{M}_{\odot}$ about Jupiter's mass. The complex CO J=6-5 spectral line shape at the location of GGTau-Aa also reveals the existence of a CO circumstellar disc of outer radius $\sim 20$ au (Methods and Extended Data: Fig.2). We do not detect cold dust emission around GGTau-Ab even though the existence of inner dust disc(s) was reported from unresolved Infrared emission ${ }^{16}$. Our $0.45 \mathrm{~mm}$ upper limits (Methods) are compatible with tidal truncation which prevents any circumstellar disc to extend beyond about $2 \mathrm{au}^{8}$. The ALMA CO J=6-5 image (Fig.1 panels a,b,c and Extended Data: Fig.1 and Fig.2) also clearly resolves $\mathrm{CO}$ gas within the central cavity with a structure indicative of the streamer-like features which have been hinted at by hydrodynamic simulations in binary systems ${ }^{5,17}$. The CO gas appears inhomogeneous as a series of fragments and the structure is dominated by an east-west extension, contrary to the very low level (Signal-to-noise ratio of 2) northern feature seen in continuum reported by ${ }^{15}$. The IRAM image (Fig.1 panels d,e,f and Extended Data: Fig.2) reveals, at lower angular resolution, that the $\mathrm{CO} J=2-1$ emission peaks are located near the inner edge of the outer ring (at radius $\sim 100-150 \mathrm{au}$ ). In contrast, the $\mathrm{CO} \mathrm{J}=6-5$ emission peaks near GGTau-Aa and GGTau-Ab, close to the bright regions of near-infrared $v=1-0 \mathrm{~S}(1) \mathrm{H}_{2}$ emission (Fig.1 panel c) interpreted as shock excited gas at the interface between the streamer and gas associated with the inner discs ${ }^{10}$. A study of the excitation conditions (Methods) reveals that CO J=2-1 and $\mathrm{J}=6-5$ emissions arise in different physical conditions. The CO J=2-1 peaks correspond to extended, cold $(\sim 35 \mathrm{~K})$ optically thick areas while the $\mathrm{CO} \mathrm{J}=6-5$ peaks trace optically thin, warmer gas $(\sim 70 \mathrm{~K})$ particularly at the interface between the streamer and the inner disc of Aa. The mass of each CO 
$\mathrm{J}=6-5$ clump is about $\sim 5 \cdot 10^{-5} \mathrm{M}_{\odot}$ (Methods). With a minimum accretion rate of $10^{-8} \mathrm{M}_{\odot} / \mathrm{yr}$, a fragment reaching the Aa disc may disappear in at most 5,000 years (a few tens times the orbital period of the binary $\mathrm{Aa}-\mathrm{Ab}$ ). The Aa disc mass currently represents about 20 such fragments; in 1 Million year, at least 200 fragments of similar mass must have been accreted to sustain such a disc. The corresponding minimum mass, that accreted from the circumbinary disc, represents about $10 \%$ of the current outer disc mass $\left(\sim 0.15 \mathrm{M}_{\odot}\right)$. The morphology of the gas in the CO J=65 and $\mathrm{J}=2-1$ maps reveals departures from symmetry, unlike hydrodynamical simulations which predict symmetric streamers for an equal-mass, low eccentricity binary system ${ }^{5}$. GGTau-Aa and $\mathrm{Ab}$ each have a mass of about $0.65 \mathrm{M}_{\odot}{ }^{13}$ and their orbital eccentricity is constrained to $e \leq 0.35^{18}$. The origin of this asymmetry might either be found in this eccentricity ${ }^{19,20}$ or in the triple nature of GGTau, as the binarity of Ab breaks the symmetry.

The change in velocity of the $\mathrm{CO} \mathrm{J}=6-5$ emission along the major axis of the dust ring is similar to that of CO J=2-1 (Fig.1 panels b,e and Extended Data: Fig.1 and 2) and the velocity gradient is that of a rotating disc ${ }^{14,21}$. At radius $200 \mathrm{au}$, we find that the velocity of the $\mathrm{CO} \mathrm{J}=6-5$ gas agrees with the known Keplerian speed (Methods and Extended Data: Table 1) derived from existing ${ }^{13} \mathrm{CO}$ maps ${ }^{14,21}$ and corresponds to the canonical dynamical mass of the triple star GGTau$\mathrm{A}\left(1.28 \mathrm{M}_{\odot}\right)^{14,21}$. This is still true down to a radius of $\sim 70-80 \mathrm{au}$. Closer to the stars, the velocity pattern of the $\mathrm{CO} \mathrm{J}=6-5$ becomes dominated by the individual gravitational field of GGTau-Aa and GGTau-Ab. Limited angular resolution (35 au) precludes detailed kinematical analysis but the study of northern $\mathrm{CO} \mathrm{J}=6-5$ peak vicinity reveals that its velocity gradient is dominated by rotation, with departure compatible with infall motion (Methods and Extended Data: Fig.3). 
A second result deals with the dust and gas circumbinary reservoir. The dust emission from the circumbinary ring is very well resolved (Fig.1 and Extended Data: Fig.4) but appears uniform within the noise. The combination of the $0.45 \mathrm{~mm}$ ALMA image with existing 1.3 and $3.4 \mathrm{~mm}$ continuum maps ${ }^{14,21}$ allows for a direct measurement of the radial dependance of the temperature in the circumbinary dust disc. Using a radiative transfer model (Methods), we determined the dust temperature profile $T_{D}(r)=13.8 \times(r / 200 \mathrm{au})^{-1.1} \mathrm{~K}$ which gives a temperature of $\sim 8.5 \mathrm{~K}$ at $300 \mathrm{au}$. Thanks to the $0.45 \mathrm{~mm}$ image, the derived dust temperature is almost independent from the dust properties. This represents a significant improvement on the knowledge of the midplane physical conditions (Methods, Extended Data: Table 2 and Fig.4). Since our measurement is the result of vertical averaging of the temperature and the upper layers of the disc are hotter due to direct irradiation by the stellar UV flux, the mid-plane must be even colder. The limited grain growth (Methods) helps explaining the low temperature since small grains are most efficient at attenuating UV radiation coming from the central stars. Furthermore, at the ring inner edge, a puffed-up rim, caused by direct stellar heating, should cast a shadow on the outer region of the disc. Such a shielding effect could explain the steep slope of the temperature profile. Previous ${ }^{13} \mathrm{CO}$ measurements and analysis yielded a similarly steep slope (exponent $\sim 0.9$ ) for the gas temperature profile in the circumbinary disc but a warmer gas temperature $(\sim 20 \mathrm{~K} \text {, at } 300 \mathrm{au})^{21}$. This is consistent with $\mathrm{CO}$ molecules being trapped onto grains in the mid-plane, whose temperature is below the $\mathrm{CO}$ freeze-out point $(\sim 17 \mathrm{~K})$, and $\mathrm{CO}$ gas only present in the heated upper layers of the $\operatorname{disc}^{22}$.

Fig. 1 shows that, unlike the continuum emission of dust, little $\mathrm{CO} \mathrm{J}=6-5$ line emission is 
detected near the circumbinary disc inner radius at $\sim 200-250$ au. This could be attributable to both inadequate $\mathrm{H}_{2}$ density to thermalize the upper energy level of the $\mathrm{CO}$ transition and to lower sensitivity of the interferometer to relatively large angular scale ( $\left.>1^{\prime \prime}\right)$ structure (Methods). Fig. 1 also shows strong excess of CO J=6-5 and 2-1 emission at position angle $\sim 120^{\circ}$ (measured East of North) and radius $\sim 240-270$ au. From these CO data alone we cannot discriminate between a temperature and/or density increase (Methods). We suggest that this localized emission is attributable to a progenitor of a sub-stellar body reminiscent of the planetary-mass companion imaged around the low-mass binary $2 \mathrm{MASS} 0103 \mathrm{AB}^{23}$. Such a companion would provide a natural explanation for the confinement of $\sim 80 \%$ of the circumbinary mass within the $\sim 90$ au breadth of the ring ${ }^{21}$. Simulations of the evolution of binary systems in which Kepler detected exoplanets demonstrate that Saturn-mass proto-planets can remain at the ring outer edge in some $\operatorname{cases}^{20}$. We do not detect an outer gap in the gas and dust circumbinary disc of GG Tau-A but existing ${ }^{12} \mathrm{CO}$, ${ }^{13} \mathrm{CO}$ and thermal dust images are not incompatible with its existence (Methods).

Our observations demonstrate that active replenishment from the outer disk can sustain the circumprimary disc surrounding GGTau-Aa beyond accretion lifetime, increasing its potential for planet formation. The presence of the condensation at the inner edge of the outer ring is puzzling and needs further investigations to determine its links with accretion processes and possible planet formation. Since almost half of Sun-like stars were born in multiple systems ${ }^{24}$, our observations provide a step towards understanding the true complexity of protoplanetary discs in multiple stellar systems and unveiling planet formation mechanisms for a significant fraction of stellar systems in our Galaxy. 


\section{References}

1. Nelson, A. F. Planet Formation is Unlikely in Equal-Mass Binary Systems with A 50 AU. Astrophys. J. Letters 537, L65-L68 (2000). astro-ph/ 0005451.

2. Mayer, L., Wadsley, J., Quinn, T. \& Stadel, J. Gravitational instability in binary protoplanetary discs: new constraints on giant planet formation. Mon. Not. R. Astron. Soc. 363, 641-648 (2005). astro-ph/0405502.

3. Thébault, P., Marzari, F. \& Scholl, H. Relative velocities among accreting planetesimals in binary systems: The circumprimary case. Icarus 183, 193-206 (2006). ast ro-ph / 0602046.

4. Artymowicz, P. \& Lubow, S. H. Dynamics of binary-disk interaction. 1: Resonances and disk gap sizes. Astrophys. J. 421, 651-667 (1994).

5. Bate, M. R. \& Bonnell, I. A. Accretion during binary star formation - II. Gaseous accretion and disc formation. Mon. Not. R. Astron. Soc. 285, 33-48 (1997).

6. Casassus, S. et al. Flows of gas through a protoplanetary gap. Nature 493, 191-194 (2013). 1305.6062

7. Skrutskie, M. F. et al. Detection of circumstellar gas associated with GG Tauri. Astrophys. J. 409, 422-428 (1993).

8. Di Folco, E. et al. GG Tauri: the fifth element. Astron. Astrophys. 565, L2 (2014). 1404.2205. 
9. Andrews, S. M. et al. Resolved Multifrequency Radio Observations of GG Tau. ArXiv e-prints (2014). 1404.5652.

10. Beck, T. L. et al. Circumbinary Gas Accretion onto a Central Binary: Infrared Molecular Hydrogen Emission from GG Tau A. Astrophys. J. 754, 72 (2012). 1205.1526.

11. Guilloteau, S. \& Dutrey, A. GG Tau: The Ringworld Revisited. In Zinnecker, H. \& Mathieu, R. (eds.) The Formation of Binary Stars, vol. 200 of IAU Symposium, 229 (2001).

12. Hartigan, P. \& Kenyon, S. J. A Spectroscopic Survey of Subarcsecond Binaries in the TaurusAuriga Dark Cloud with the Hubble Space Telescope. Astrophys. J. 583, 334-357 (2003). astro-ph/0209608.

13. White, R. J., Ghez, A. M., Reid, I. N. \& Schultz, G. A Test of Pre-Main-Sequence Evolutionary Models across the Stellar/Substellar Boundary Based on Spectra of the Young Quadruple GG Tauri. Astrophys. J. 520, 811-821 (1999). astro-ph/9902318.

14. Dutrey, A., Guilloteau, S. \& Simon, M. Images of the GG Tauri rotating ring. Astron. Astrophys. 286, 149-159 (1994).

15. Piétu, V., Gueth, F., Hily-Blant, P., Schuster, K.-F. \& Pety, J. High resolution imaging of the GG Tauri system at 267 GHz. Astron. Astrophys. 528, A81 (2011). 1102.4029.

16. Skemer, A. J. et al. Dust Grain Evolution in Spatially Resolved T Tauri Binaries. Astrophys. J. 740, 43 (2011). 1107.3161. 
17. Artymowicz, P., Clarke, C. J., Lubow, S. H. \& Pringle, J. E. The effect of an external disk on the orbital elements of a central binary. Astrophys. J. Letters 370, L35-L38 (1991).

18. Beust, H. \& Dutrey, A. Dynamics of the young multiple system GG Tauri. I. Orbital fits and inner edge of the circumbinary disk of GG Tau A. Astron. Astrophys. 439, 585-594 (2005).

19. Pierens, A. \& Nelson, R. P. On the migration of protoplanets embedded in circumbinary disks. Astron. Astrophys. 472, 993-1001 (2007). 0707.2677.

20. Pierens, A. \& Nelson, R. P. Migration and gas accretion scenarios for the Kepler 16, 34, and 35 circumbinary planets. Astron. Astrophys. 556, A134 (2013). 1307.0713.

21. Guilloteau, S., Dutrey, A. \& Simon, M. GG Tauri: the ring world. Astron. Astrophys. 348, 570-578 (1999).

22. Dutrey, A. et al. Physical and chemical structure of planet-forming disks probed by millimeter observations and modeling. ArXiv e-prints (2014). 1402.3503.

23. Delorme, P. et al. Direct-imaging discovery of a 12-14 Jupiter-mass object orbiting a young binary system of very low-mass stars. Astron. Astrophys. 553, L5 (2013). 1303 . 4525.

24. Duchêne, G. \& Kraus, A. Stellar Multiplicity. Annual Rev. Astron. Astrophys. 51, 269-310 (2013). 1303.3028.

Acknowledgements ALMA is a partnership of ESO (representing its member states), NSF (USA) and NINS (Japan), together with NRC (Canada) and NSC and ASIAA (Taiwan), in cooperation with the Republic of Chile. The Joint ALMA Observatory is operated by ESO, AUI/NRAO and NAOJ. IRAM is supported 
by INSU/CNRS (France), MPG (Germany) and IGN (Spain). A.D. thanks the French programs PNP, PCMI, PNPS and ASA for providing fundings for this study.

Authors Contributions A.D.led the project and participated in data reduction. All authors contributed to the data analysis, discussed the results and commented on the manuscript.

Competing Interests The authors declare that they have no competing financial interests.

Correspondence Correspondence and requests for materials should be addressed to Anne Dutrey (email: Anne.Dutrey@obs.u-bordeaux1.fr).

This paper makes use of the following ALMA data: ADS/JAO.ALMA2011.0.00059. 
Figure 1: ALMA (a,b,c) and IRAM (d,e,f) GGTau-A images. (a) $0.45 \mathrm{~mm}$ emission (contours) and CO 6-5 flux (color). (b) $0.45 \mathrm{~mm}$ emission and $\mathrm{CO}$ velocity field (color). (c) $0.45 \mathrm{~mm}$ emission (color) with $\mathrm{CO}$ 6-5 flux (blue contours) and $\mathrm{H}_{2}$ intensity (red contours). (d,e) as (a,b) but for $1.3 \mathrm{~mm}$ emission (contours) and CO 2-1 flux (color), (f) $1.3 \mathrm{~mm}$ emission (color) and $\mathrm{H}_{2}$ intensity (contours). Positions are relative to R.A. $=$ 04:32:30.359 and Dec. $=17: 31: 40.38$ (J2000). Crosses are the locations of $\mathrm{Aa}$ and $\mathrm{Ab}$ components, triangles and squares the locations of the $\mathrm{CO} \mathrm{J}=6-5$ and $\mathrm{J}=2-1$ peaks, respectively. Wedge units are $\mathrm{Jy} / \mathrm{beam} . \mathrm{km} / \mathrm{s}$ in $(\mathrm{a}, \mathrm{d}), \mathrm{km} / \mathrm{s}$ in $(\mathrm{b}, \mathrm{e})$ and $\mathrm{mJy} / \mathrm{beam}$ in $(\mathrm{c}, \mathrm{f})$.

\section{Methods}

ALMA Observations. GGTau was observed with ALMA in Cycle 0 (project 2011.0.00059.S) in Band 9 on August 13 2012. The spectral setup was of 4 spectral windows of total width 0.938 $\mathrm{GHz}$, centered at $691.485,689,676$ and $674 \mathrm{GHz}$ respectively. The correlator observed in dual polarization, yielding a channel spacing of $244 \mathrm{kHz}$, or $0.11 \mathrm{~km} / \mathrm{s}$. With Hanning smoothing, the spectral resolution is twice larger than those values. The array of 23 antennas was in its extended configuration. The water vapor was about $0.25 \mathrm{~mm}$ and system temperatures ranged from 500 to $1000 \mathrm{~K}$. Comparison of the $\mathrm{CO}$ data with existing ${ }^{12} \mathrm{CO} \mathrm{J}=2-1$ IRAM data (Fig.1) revealed a frequency shift of the ALMA observations whose origin could not be identified. We corrected the velocity offset by correlating with the IRAM data. The velocity difference was $0.97 \pm 0.01 \mathrm{~km} / \mathrm{s}$. Since it is an offset, it does not introduce any error on the velocity gradient. The absolute flux scale 
was determined using Ceres. The ALMA data were calibrated using CASA then exported through UVFITS to be imaged and analysed using GILDAS. Extended Data: Fig.1 presents the resulting channel maps.

The integrated ring+disc continuum flux is in good agreement with the previous unresolved detection $^{25}$ : no more than $20 \%$ of the flux is lost.

For the CO J=6-5 line, we only recover a fraction of the integrated line flux of $\sim 38 \pm 8$ Jy.km/s ${ }^{26}$. The ALMA antenna configuration exhibits a lack of short baselines which induces a partial loss of flux for the extended structures that deconvolution cannot recover, particularly in the $\mathrm{CO}$ circumbinary disc which extends up to $800 \mathrm{au}$. We ran several disc simulations of the $\mathrm{CO}$ $\mathrm{J}=6-5$ emission using the ALMA simulator ${ }^{27}$. If emission only arises from the ring, our simulation shows that about $20 \%$ of the emission would be filtered out by the configuration of ALMA. A much larger fraction is lost if CO J=6-5 extends in the whole outer disc $\left(\mathrm{R}_{\text {out }}=800 \mathrm{au}\right)$, this fraction depends on signal to noise. The expected extension of the $\mathrm{CO} \mathrm{J}=6-5$ emission depending a priori on the excitation conditions in the outer disc, uncertainties on the amount of flux loss preclude any quantitative analysis of the large scale disc emission in $\mathrm{CO} \mathrm{J}=6-5$ data.

IRAM Data We complement ALMA data with $\mathrm{CO} J=2-1$ and continuum images from the IRAM interferometer. The compact configuration was observed on Dec 5/6 1997, and long baselines up to $400 \mathrm{~m}$ were obtained in Jan 28-31, 1998 and Mar 18, $1998^{11}$. The unpublished extended configuration data were obtained on Feb, 16 2008. System temperatures were 120 K. Flux calibration, based on MWC349, gives an absolute flux accuracy of about 10\%. Imaging was performed using 
the GILDAS software. The resulting beam size is $0.65^{\prime \prime} \times 0.29^{\prime \prime}$ at PA $21^{\circ}$. The spectral resolution is $0.10 \mathrm{~km} / \mathrm{s}$, and the effective noise about $14 \mathrm{mJy} / \mathrm{beam}$ (up to $20 \mathrm{mJy} / \mathrm{beam}$ on the channels with the most extended emission due to deconvolution limits). The continuum image at $1.3 \mathrm{~mm}$ was produced using line-free channels. Its effective noise is limited by dynamic range to about 1 mJy/beam. Extended Data: Fig.2 shows the CO J=2-1 channel maps.

Proper Motions. Accounting for the expected orbital motion, we adopted proper motions of (17,19) mas/yr for the center of mass of the system ${ }^{28}$ to merge all data.

Inner dust disc properties For the disc of GGTau-Aa, the total fluxes of $55 \mathrm{mJy}$ at $0.45 \mathrm{~mm}$ and $10 \mathrm{mJy}$ at $1.3 \mathrm{~mm}$ are consistent with an optically thick dust emission at mean temperature $\sim$ $35 \mathrm{~K}^{29}$ and an outer radius of the order of $\sim 7 \mathrm{au}$, a size in agreement with the tidal truncation by GGTau-Ab. Assuming a standard dust opacity of $0.02 \mathrm{~cm}^{2} \mathrm{~g}^{-1}$ at $1.3 \mathrm{~mm}$, the minimum disc mass is roughly $10^{-3} \mathbf{M}_{\odot}$ or a Jupiter's mass worth of gas and dust. The rate at which matter is accreting from the disc onto the surface of GGTau-Aa is in the range of $\sim 10^{-7.56}-10^{-8.02} \mathrm{M}_{\odot} / \mathrm{yr}^{12}$. Such a disc would dissipate in less than $\sim 3 \cdot 10^{4}-10^{5}$ yr without external replenishment. We place an upper limit on the circumstellar disc mass surrounding GGTau-Ab of $\sim 10^{-5} \mathrm{M}_{\odot}$ at $3 \sigma$ (a little more than one Earth mass) or an upper limit on its radius of $\sim 1$ au by extrapolating the dust opacity to $0.45 \mathrm{~mm}, 0.067 \mathrm{~cm}^{2} \mathrm{~g}^{-1}$, and using a similar temperature as in the disc of GGTau-Aa. This is consistent with the separation of $\mathrm{Ab} 1$ and $\mathrm{Ab} 2$ of $\sim 4.5 \mathrm{au}^{8}$.

Inner cavity: CO excitation conditions We performed escape probability calculations using the code $\mathrm{RADEX}^{30}$ (http://www.sron.rug.nl/ vdtak/radex/radex.php), a radiative transfer code dedi- 
cated to calculations of molecular line excitations. At the location of the eastern $\mathrm{CO} J=2-1$ peak ( $\sim 80$ au from the stars), the CO lines are optically thick, $\mathrm{H}_{2}$ densities must be greater than $10^{5} \mathrm{~cm}^{-3}$, and the gas is cold with a temperature in the range $\sim 30-40 \mathrm{~K}$. Closer to the stars, the peaks of the bright $\mathrm{CO} \mathrm{J}=6-5$ regions correspond to optically thin emission, with a warmer temperature of about $70 \mathrm{~K}$ and $\mathrm{H} \mathrm{H}_{2}$ density of $\sim 5 \cdot 10^{5} \mathrm{~cm}^{-3}$. These densities agree with the preshock gas densities derived from shock models for the $2.12 \mu \mathrm{m} \mathrm{H}_{2}$ emission maps. With a standard $\mathrm{CO} / \mathrm{H}_{2}$ ratio of $10^{-4}$, the mass of each optically thin clump is of the order of $\sim 3-7 \cdot 10^{-5} \mathrm{M}_{\odot}$.

Inner cavity: $\mathrm{CO}$ velocity field We studied the velocity variations of the $\mathrm{CO} \mathrm{J}=6-5$ emission. The spectra displayed in Fig.3 (Extended Data), at the locations of the CO J=6-5 clumps also show that the velocity is dominated by rotation. The complexity of the system which is triple and the quality of the data precludes any detailed modeling of the velocity field but some points can be addressed. From a compilation of existing values in the literature, we derive for GGTau-Aa, a velocity of $5.8 \pm 0.2 \mathrm{~km} / \mathrm{s}^{31}$. This implies a difference of the order of $0.6 \pm 0.2 \mathrm{~km} / \mathrm{s}$ with the known systemic (barycentric) velocity of the whole stellar system ${ }^{21}$. Fig.3 (Extended Data) reveals that the measured CO gas velocity near GGTau-Aa is in very good agreement with Aa velocity as it is expected for bound motions. Around the disc minor axis, we note that the iso-velocity contours are twisted at a radius of about 70-80 au from the mass center. Inside this radius, the iso-contours are closely packed, and suddenly spread out beyond. Furthermore, the iso-velocity contours are aligned on the Aa-Ab line between GGTau Aa and Ab. This is exactly what is expected when the gravitation field, externally dominated by the total stellar mass $\left(1.28 \mathrm{M}_{\odot}\right)$ becomes dominated by the individual objects Aa and the binary Ab. At the location of the $\mathrm{CO} J=6-5$ clumps, the spectra 
displayed in Fig. 3 also exhibit a large velocity dispersion ( $\delta v \simeq 2-2.5 \mathrm{~km} / \mathrm{s})$ which can be partly due to infall. We check this by analysing the velocity field along the minor axis at the northern clump. In an inclined disc, projected velocities due to infall are maximum along the minor axis contrary to rotation velocities which are maximum along the major axis ${ }^{32,33}$. Taking into account the beam size of $35 \mathrm{au}$, we find that the maximum velocity dispersion along the minor axis in a spectrum would be of the order $1.2 \mathrm{~km} / \mathrm{s}$ if it is only due to Keplerian rotation around central objects of $0.65 \mathrm{M}_{\odot}$ (because this CO J=6-5 clump is located in the area where the velocity field is dominated by GGTau-Ab). This is a factor two lower than the observed line-widths of $2-2.5 \mathrm{~km} / \mathrm{s}$. In discs, turbulence cannot explain such a large dispersion ${ }^{34}$. However, infall velocities can be $\sqrt{2}$ larger than Keplerian velocities. It is therefore reasonable to conclude that the observed velocities and line-widths are compatible with a combination of rotation and infall. For the southern clump, located near GGTau-Aa, the velocity profile is very complex. For instance, the spectrum onto the Aa star shows two double peaked profiles, perfectly centered on the velocity of Aa $(5.8 \mathrm{~km} / \mathrm{s})$, but with significantly different widths $(F W H M \simeq 1.5$ and $6 \mathrm{~km} / \mathrm{s})$. The total velocity dispersion of the broader double peaked profile $(\sim 6 \mathrm{~km} / \mathrm{s})$ is compatible with a circumstellar disc of outer radius of the order of $\sim 20 \mathrm{au}^{35}$, if the inclination of the system is the same as that of the outer ring $\left(35^{\circ}\right)$. Such a CO outer radius is compatible with the value derived from the dust (provided a small part of the dust emission is optically thin) and tidal truncation due to GGTau-Ab (the physical star separation being about $42 \mathrm{au}$ ).

Outer disc: dust ring properties We use Diskfit, a radiative transfer code dedicated to the modeling of the disc images in $\mathrm{mm} / \mathrm{submm}$ regime ${ }^{36}$, to analyse the dust properties inside the outer 
ring using the best data available ( $3 \mathrm{~mm}$ and $1.3 \mathrm{~mm}$ PdBI data and $0.45 \mathrm{~mm}$ ALMA observations). A modified Levenberg-Marquardt scheme with step adjustment is used to derive the best fit ${ }^{37}$. The analysis is performed in the Fourier plane to avoid non linear effects due to the deconvolution. We explore large ranges for all parameters to avoid local mimima. Fig.4 and Table 2 (Extended Data) present the results, with $1 \sigma$ formal errorbars derived from the covariance matrix. The errors are computed on the basis of the difference between data and model, so includes dynamic range limitations in addition to thermal noise. We use simple truncated power laws to model the surface density and temperature. We allow dust emissivity to vary with frequency as $\left(\kappa_{\nu}=\kappa_{o} \times\left(\nu / \nu_{o}\right)^{\beta}\right)$. Such a simple model is valid for the dust distribution since the emission is known to be confined in a ring with sharp edges ${ }^{9,21}$. The derived geometrical parameters are in very good agreement with the previous results ${ }^{21}$. Since the dust temperature is mostly constrained by the ratio of the flux density at $0.45 \mathrm{~mm}$ and $1.3 \mathrm{~mm}$, we checked that the results remain robust even assuming an absolute flux calibration error as high as $20 \%$ at $0.45 \mathrm{~mm}$. The density, temperature and dust spectral index are robust with respect to all minimizations. However, we find a slight dependence between $\beta$ and the surface density. This is not surprising because in the optically thin case, the brightness temperature is proportional to $\tau \propto \kappa(0.45 \mathrm{~mm}) \times \Sigma(r)$ (with $\kappa(0.45 \mathrm{~mm})$ the absorption coefficient of the dust at $0.45 \mathrm{~mm}$ ). A better removal of this dependence would require higher angular resolution at $1.3 \mathrm{~mm}$. We found a value for the spectral index $\beta=1.0 \pm 0.1$, which indicates moderate grain growth compared to interstellar dust. Such a grain growth is compatible with a recent analysis of the dust ring performed between 7 and $1.3 \mathrm{~mm}^{9}$. We found a marginal dependency of $\beta$ with radius, suggesting that larger dust particles reside near the inner edge of the outer disc (i.e. closer 
to the central stars), as observed for some circumstellar discs orbiting young single stars ${ }^{38}$. So far, dust temperatures were derived either from Monte-Carlo dust disc models ${ }^{39}$ or one-dimensional thermal models associated to pure $\mathrm{mm}$ data $^{9}$. In our case, we are able to directly derive the dust temperature thanks to the deviation from the Rayleigh Jeans regime at $0.45 \mathrm{~mm}$, combined with sufficiently high spatial resolution at $0.45 \mathrm{~mm}$ and $1.3 \mathrm{~mm}$. This permits to remove the degeneracy between the temperature and the opacity. This direct measurement is of great interest for a better characterisation of disc mid-planes where planet formation is expected to occur.

Outer disc: CO excitation conditions Analysis of the physical conditions in the outer ring at radius around 200 au shows that the mid-plane $\mathrm{H}_{2}$ density $\left(\sim 10^{9} \mathrm{~cm}^{-3}\right)$ is high enough to thermalise $\mathrm{CO}$ transitions up to $\mathrm{J}=6-5^{21}$. Excitation of the $\mathrm{CO} \mathrm{J}=6-5$ line requires a $\mathrm{H}_{2}$ critical density of $\sim 3 \cdot 10^{5} \mathrm{~cm}^{-3}$. We also derive a dust temperature lower than the $\mathrm{CO}$ freeze out temperature of $17 \mathrm{~K}$. Therefore the observed large scale emissions from the CO $\mathrm{J}=1-0$ and $\mathrm{J}=2-1$ transitions must arise from a warmer molecular layer located above the mid-plane as predicted by layered disc models ${ }^{22}$. and revealed by ALMA observations of HD $163296^{40}$.

Using our measurement of the dust temperature we find that the hydrostatic scale height of the gas and dust is about $\sim 25$ and $\sim 35$ au at radii of 200 and 300 au, respectively. If the CO gas originates in a layer located at about 3 scale heights (in agreement with chemical models), and taking into account the vertical temperature gradient the $\mathrm{H}_{2}$ density would be $\sim 10^{5}$ and $\sim 10^{4} \mathrm{~cm}^{-3}$ at radii of 200 and 300 au, respectively. This is sufficient to thermalise the $\mathrm{J}=1-0$ and $\mathrm{J}=2-1$ lines, but not the $\mathrm{J}=6-5$ transition, which can be partially sub-thermally excited outside the ring. Note that any gap at the outer edge of the ring created by an embedded proto-planet (see 
section below) would imply some sub-thermal excitation there.

Outer disc: a CO hot spot at the outer edge of the ring The $\mathrm{CO} J=6-5$ and $\mathrm{J}=2-1$ maps reveal a brightness enhancement of unknown origin near the outer edge of the dust ring (Fig.1 and spectrum in Extended Data: Fig.3). From the CO J=2-1 emission which is optically thick and thermalised, we derive a minimum temperature of $\sim 40 \mathrm{~K}$ in this clump. This indicates a temperature enhancement of at least $20 \mathrm{~K}$ compared to the surrounding optically thick $\mathrm{CO}$ gas. The feature is clearly dominated by the gas emission, unlike the recent detection of a dusty vortex-like structure, or dust trap, in the circumstellar disc associated with Oph IRS $48^{41}$. In the high angular resolution continuum maps at $0.45 \mathrm{~mm}$ and $1.3 \mathrm{~mm}$, there is no dust counterpart to this bright $\mathrm{CO}$ peak. The dust absorption coefficient being small, only a sufficiently large and massive dust clump would be detectable. Given the effective (dynamic range limited) noise, the (dust) mass limit is about a few $10^{-7} \mathbf{M}_{\odot}$ at $3 \sigma$, or the size must be smaller than about 4 au, assuming a dust temperature of 40 K. In Fig.3 (Extended Data), there is a marginal excess emission at $1.3 \mathrm{~mm}$ ( $4 \sigma$ level) in the difference map (right panel) at the location of the blob. This has to be taken with caution since there are other $4 \sigma$ peaks which likely trace some extended cold emission beyond the ring outer radius ${ }^{21}$. Analysis of the GGTau-A dust ring in term of dust trap, based on resolved images between 7 and $1.3 \mathrm{~mm}$, have been recently achieved but remains inconclusive ${ }^{9}$. The presence of the outer binary GGTau-B could induce gravitational disturbances in the circumbinary disc especially if its orbit is eccentric $^{42}$. However, the total mass of GG Tau B is very low $\left(\sim 0.17 \mathrm{M}_{\odot}{ }^{13}\right)$ and it is located far away from the GGTau-A circumbinary ring ( 10" or $1400 \mathrm{au})$. Therefore, we conclude that it is unlikely that GG Tau B would have induced such a sizeable disturbance in the circumbinary ring. 
We explore here another interesting possibility; this feature would be an indirect evidence for an unknown (already formed) embedded companion that is still actively accreting material from the circumbinary $\operatorname{disc}^{43}$. In this scenario, the planet remains undetectable at mm wavelengths but the warmer accreting envelope can be seen ${ }^{44,45}$. The presence of such a companion would provide an explanation for the mass confinement in a narrow ring $(\sim 80 \%$ of the circumbinary mass is located within the $\sim 90$ au breadth ring ${ }^{21}$ ). This situation is similar to the gas free, dusty ring orbiting the more evolved star Fomalhaut, where the confinement by shepherding planets appears as the most consistent explanation ${ }^{46}$.

Simulations of the evolution of Kepler systems where exoplanets have been observed reveal that Saturn-like planets are expected to migrate to reach the 4:1 resonance ${ }^{20}$ without opening a gap, except if the disc is very cold, as is the case for the GGTau ring. In such cold discs, a proto-planet can remain at the ring outer edge and the size of the gap will mainly depend on the planet mass ${ }^{20}$. So far, we do not detect a gap in the gas and dust circumbinary distribution of GGTau-A. Our CO $\mathrm{J}=6-5$ map shows that there is almost no $\mathrm{CO} \mathrm{J}=6-5$ emission located outside the outer ring; this is expected if there is a gap or a strong density decrease which implies sub-thermal excitation. Gap opening depends on several physical parameters such as the mass of the planet, the disc viscosity and aspect ratio ${ }^{47}$. For example, a Saturn-like body located at $250 \mathrm{au}$, in the circumbinary disc would open a gap of about 35 au assuming standard viscosity $\left(\alpha=10^{-2}\right)$ and aspect ratio $\mathrm{h}(\mathrm{r}) / \mathrm{r}$ $=0.1$. Under the same physical conditions, the gap would be only 12 au wide for a Neptune-like planet $^{48}$. Observing a gap in the existing CO J=2-1 map would be difficult because the CO $\mathrm{J}=2-1$ line is very optically thick, making the expected ring/gap brightness contrast difficult to detect, 
except if the gap is very deep with a high ring/gap density contrast (larger than the CO opacity), and is large enough to be directly seen (about 40 au compared to the beam size). ${ }^{13} \mathrm{CO}$ is less optically thick (by a factor 70 ) but the existing ${ }^{13} \mathrm{CO} \mathrm{J}=2-1$ map ${ }^{21}$ has an angular resolution $(0.88 \times$ $0.56^{\prime \prime}$ or $120 \times 80 \mathrm{au}$ ) insufficient for a direct imaging. However, a ring/outer disc density contrast of $\sim 25$ at 260 au was reported ${ }^{21}$. This contrast can be the signature of an unresolved gap in the ${ }^{13} \mathrm{CO} \mathrm{J}=2-1$ map. A spectroscopic detection ${ }^{49}$ would be possible only on very high sensitivity data which does not exist so far. Very sensitive observations of optically thinner CO isotopologues at high angular resolution ( $\left.\ll 0.1-0.2^{\prime \prime}\right)$ are needed to study the clump properties (including viewing of a gap) and its implications for planet formation scenarios.

\section{References}

25. Moriarty-Schieven, G. H. \& Butner, H. M. A Submillimeter-Wave "Flare" from GG Tauri? Astrophys. J. 474, 768-773 (1997). astro-ph/9607159.

26. Thi, W.-F., van Dishoeck, E. F., Blake, G. A., van Zadelhoff, G.-J. \& Hogerheijde, M. R. Detection of H_2 Pure Rotational Line Emission from the GG Tauri Binary System. Astrophys. J. Letters 521, L63-L66 (1999). astro-ph/9906231.

27. Pety, J., Gueth, F. \& Guilloteau, S. ALMA+ACA Simulation Tool. ALMA Memo 386, 1-10 (2002)

28. Ducourant, C. et al. Pre-main sequence star Proper Motion Catalogue. Astron. Astrophys. 438, 769-778 (2005). 
29. Piétu, V., Guilloteau, S., Di Folco, E., Dutrey, A. \& Boehler, Y. Faint disks around classical T Tauri stars: small but dense enough to form planets. Astron. Astrophys. 564, A95 (2014).

30. van der Tak, F. F. S., Black, J. H., Schöier, F. L., Jansen, D. J. \& van Dishoeck, E. F. A computer program for fast non-LTE analysis of interstellar line spectra. With diagnostic plots to interpret observed line intensity ratios. Astron. Astrophys. 468, 627-635 (2007). 0704.0155.

31. Nguyen, D. C., Brandeker, A., van Kerkwijk, M. H. \& Jayawardhana, R. Close Companions to Young Stars. I. A Large Spectroscopic Survey in Chamaeleon I and Taurus-Auriga. Astrophys. J. 745, 119 (2012). 1112.0002.

32. Guilloteau, S. \& Dutrey, A. Physical parameters of the Keplerian protoplanetary disk of DM Tauri. Astron. Astrophys. 339, 467-476 (1998).

33. Rosenfeld, K. A., Chiang, E. \& Andrews, S. M. Fast Radial Flows in Transition Disk Holes. Astrophys. J. 782, 62 (2014). 1312.3817.

34. Guilloteau, S. et al. Chemistry in disks. VIII. The CS molecule as an analytic tracer of turbulence in disks. Astron. Astrophys. 548, A70 (2012). 1211.4969.

35. Beckwith, S. V. W. \& Sargent, A. I. Molecular line emission from circumstellar disks. Astrophys. J. 402, 280-291 (1993).

36. Boehler, Y., Dutrey, A., Guilloteau, S. \& Piétu, V. Probing dust settling in proto-planetary discs with ALMA. Mon. Not. R. Astron. Soc. 431, 1573-1586 (2013). 1303.5906.

37. Piétu, V., Dutrey, A. \& Guilloteau, S. Probing the structure of protoplanetary disks: a comparative study of DM Tau, LkCa 15, and MWC 480. Astron. Astrophys. 467, 163-178 (2007). astro-ph/0701425. 
38. Guilloteau, S., Dutrey, A., Piétu, V. \& Boehler, Y. A dual-frequency sub-arcsecond study of proto-planetary disks at mm wavelengths: first evidence for radial variations of the dust properties. Astron. Astrophys 529, A105 (2011). 1103.1296.

39. Madlener, D., Wolf, S., Dutrey, A. \& Guilloteau, S. The circumstellar disk of HH 30. Searching for signs of disk evolution with multi-wavelength modeling. Astron. Astrophys. 543, A81 (2012). 1205.4901.

40. de Gregorio-Monsalvo, I. et al. Unveiling the gas-and-dust disk structure in HD 163296 using ALMA observations. Astron. Astrophys. 557, A133 (2013). 1307.1357.

41. van der Marel, N. et al. A Major Asymmetric Dust Trap in a Transition Disk. Science 340, 1199-1202 (2013). 1306.1768.

42. Beust, H. \& Dutrey, A. Dynamics of the young multiple system GG Tauri. II. Relation between the stellar system and the circumbinary disk. Astron. Astrophys. 446, 137-154 (2006).

43. Gressel, O., Nelson, R. P., Turner, N. J. \& Ziegler, U. Global Hydromagnetic Simulations of a Planet Embedded in a Dead Zone: Gap Opening, Gas Accretion, and Formation of a Protoplanetary Jet. Astrophys. J. 779, 59 (2013). 1309.2871.

44. Wolf, S., Gueth, F., Henning, T. \& Kley, W. Detecting Planets in Protoplanetary Disks: A Prospective Study. Astrophys. J. Letters 566, L97-L99 (2002). a stro-ph / 0201197.

45. Wolf, S. \& D’Angelo, G. On the Observability of Giant Protoplanets in Circumstellar Disks. Astrophys. J. 619, 1114-1122 (2005). astro-ph/0 410064.

46. Boley, A. C. et al. Constraining the Planetary System of Fomalhaut Using High-resolution ALMA Observations. Astrophys. J. Letters 750, L21 (2012). 1204.0007. 
47. Crida, A., Morbidelli, A. \& Masset, F. On the width and shape of gaps in protoplanetary disks. Icarus 181, 587-604 (2006). astro-ph / 0511082.

48. Takeuchi, T., Miyama, S. M. \& Lin, D. N. C. Gap Formation in Protoplanetary Disks. Astrophys. J. 460, 832-847 (1996).

49. Dutrey, A. et al. Cavities in inner disks: the GM Aurigae case. Astron. Astrophys. 490, L15-L18 (2008).

\section{Extended Data}

Extended Data Table 1 Parameters relevant of the analysis of the ALMA data. Values are adopted from previous works.

Extended Data Table 2 Best fit results for the GG Tau circumbinary dust disc, as derived from the whole continuum data set.

Extended Data Figure 1 ALMA large scale $C O J=6-5$ channel map. The beam size is $0.29^{\prime \prime} \times 0.25^{\prime \prime}$ at P.A. $68^{\circ}$. The level step is $100 \mathrm{mJy} /$ Beam or $3.51 \mathrm{~K}$ corresponding to $3.4 \sigma$. (a) - full map. (b) - inner zoom. 
Extended Data Figure 2 Archival PdBI large scale $\mathrm{CO} J=2-1$ channel map. The beam size is $0.68^{\prime \prime} \times 0.31^{\prime \prime}$ at P.A. $21^{\circ}$. The level step is $50 \mathrm{mJy} /$ Beam or $5.48 \mathrm{~K}$ corresponding to $3.85 \sigma$. (a) - full map. (b) - inner zoom.

Extended Data Figure 3 Montage of the $\mathrm{CO} J=6-5$ data. False color: integrated area. Stars give the location of $\mathrm{Aa}$ and $\mathrm{Ab}$. Black ellipses show the ring edges. The three spectra sets (Jy/Beam unit) show the velocity gradient along the northern/southern CO $\mathrm{J}=6-5$ clump, respectively (dominated by rotation). On spectra, the red line is the systemic velocity $(6.4 \mathrm{~km} / \mathrm{s})$. From east to west, the black contours corresponds to velocity contours of $6.0,6.4$ and $6.8 \mathrm{~km} / \mathrm{s}$. The systemic velocity contour is exactly passing between the two stars (barycenter). The single spectrum corresponds to the location of the hot spot.

Extended Data Figure 4 Dust disc best model. ALMA continuum data at $0.45 \mathrm{~mm}(\mathrm{a})$. Best model at $0.45 \mathrm{~mm}$, same contour levels (b). Difference between the observations and the best model (c), contour levels corresponds to $2 \sigma$. (d,e,f): the same for the IRAM continuum data at $1.3 \mathrm{~mm}$. 

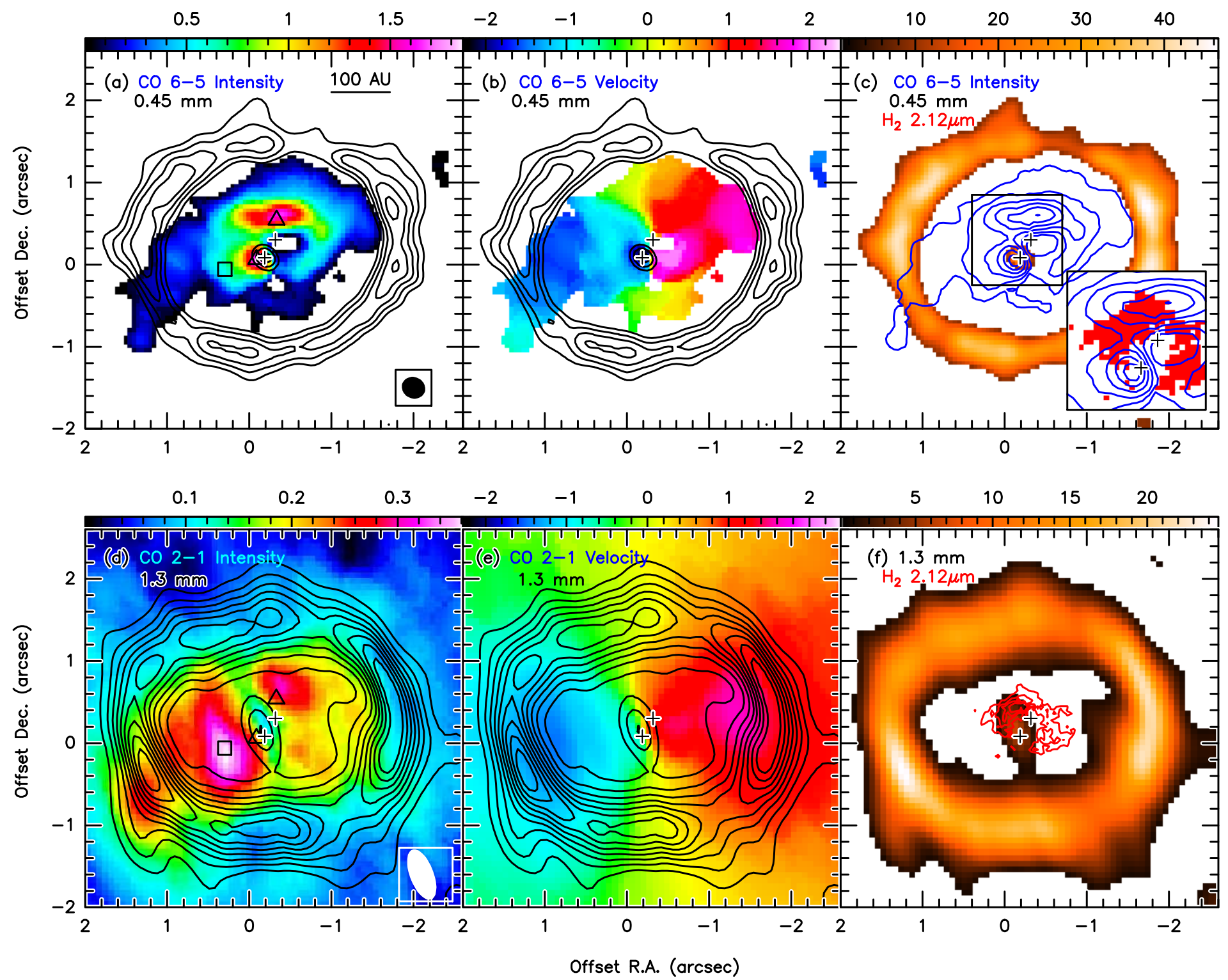


\begin{tabular}{|c|c|c|}
\hline \multicolumn{3}{|c|}{$\mathrm{CO} \mathrm{J}=2-1$ analysis } \\
\hline Systemic velocity & $V_{\mathrm{LSR}}$ & $6.38 \pm 0.02 \mathrm{~km} . \mathrm{s}^{-1}$ \\
\hline Orientation & $P A$ & $7 \pm 2^{\circ}$ \\
\hline Inclination & $i$ & $37 \pm 1^{\circ}$ \\
\hline Disk outer radius & $R_{\text {out }}$ & $\sim 800 \quad \mathrm{au}$ \\
\hline Velocity law: & \multicolumn{2}{|c|}{$V(r)=V_{100}\left(\frac{r}{100 \mathrm{au}}\right)^{-v}$} \\
\hline velocity at $100 \mathrm{au}$ & $V_{100}$ & $3.4 \pm \quad 0.1 \mathrm{~km} . \mathrm{s}^{-1}$ \\
\hline exponent & $v$ & $0.5 \pm \quad 0.1$ \\
\hline
\end{tabular}




\begin{tabular}{|c|c|c|c|}
\hline \multicolumn{4}{|c|}{ Geometry } \\
\hline Orientation & $P A$ & $6.5 \pm$ & $0.2^{\circ}$ \\
\hline Inclination & $i$ & $35.0 \pm$ & $0.2^{\circ}$ \\
\hline \multicolumn{4}{|c|}{ Size } \\
\hline Ring inner radius & $R_{i}$ & $193 \pm$ & $1 \mathrm{au}$ \\
\hline Ring outer radius & $R_{o}$ & $285 \pm$ & $1 \mathrm{au}$ \\
\hline \multicolumn{4}{|c|}{ Surface density law: $\quad \Sigma(r)=\Sigma_{200}\left(\frac{r}{200 a u}\right)^{-p}$} \\
\hline $\mathrm{H}_{2}$ surface density & $\Sigma_{200}$ & $9 \pm$ & $110^{24} \mathrm{~cm}^{-2}$ \\
\hline exponent & & $1.3 \pm$ & 0.2 \\
\hline \multicolumn{4}{|c|}{ Temperature law: $\quad T(r)=T_{200}\left(\frac{r}{200 \mathrm{au}}\right)^{-q}$} \\
\hline Dust Temperature & $T_{200}$ & $13.8 \pm$ & $0.3 \mathrm{~K}$ \\
\hline exponent & $q$ & $1.1 \pm$ & 0.1 \\
\hline Gas-to-Dust Ratio & $G / D$ & 100 & - \\
\hline Absorption Coefficient (gas+dust) & $\kappa(1.3 \mathrm{~mm})$ & $0.02 \mathrm{~cm}^{2} / \mathrm{g}$ & - \\
\hline Dust Spectral Index & $\beta$ & $1.0 \pm$ & 0.1 \\
\hline
\end{tabular}



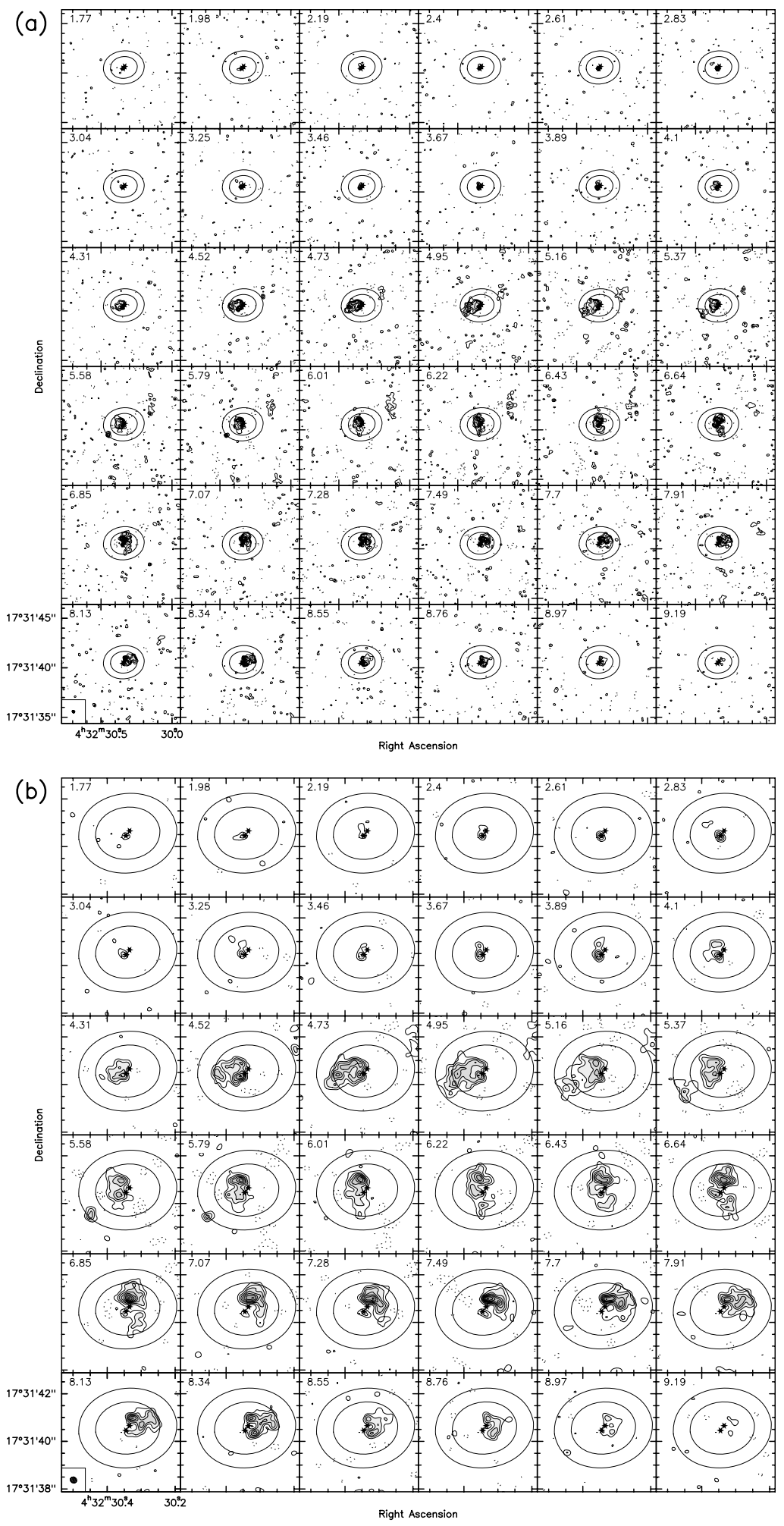

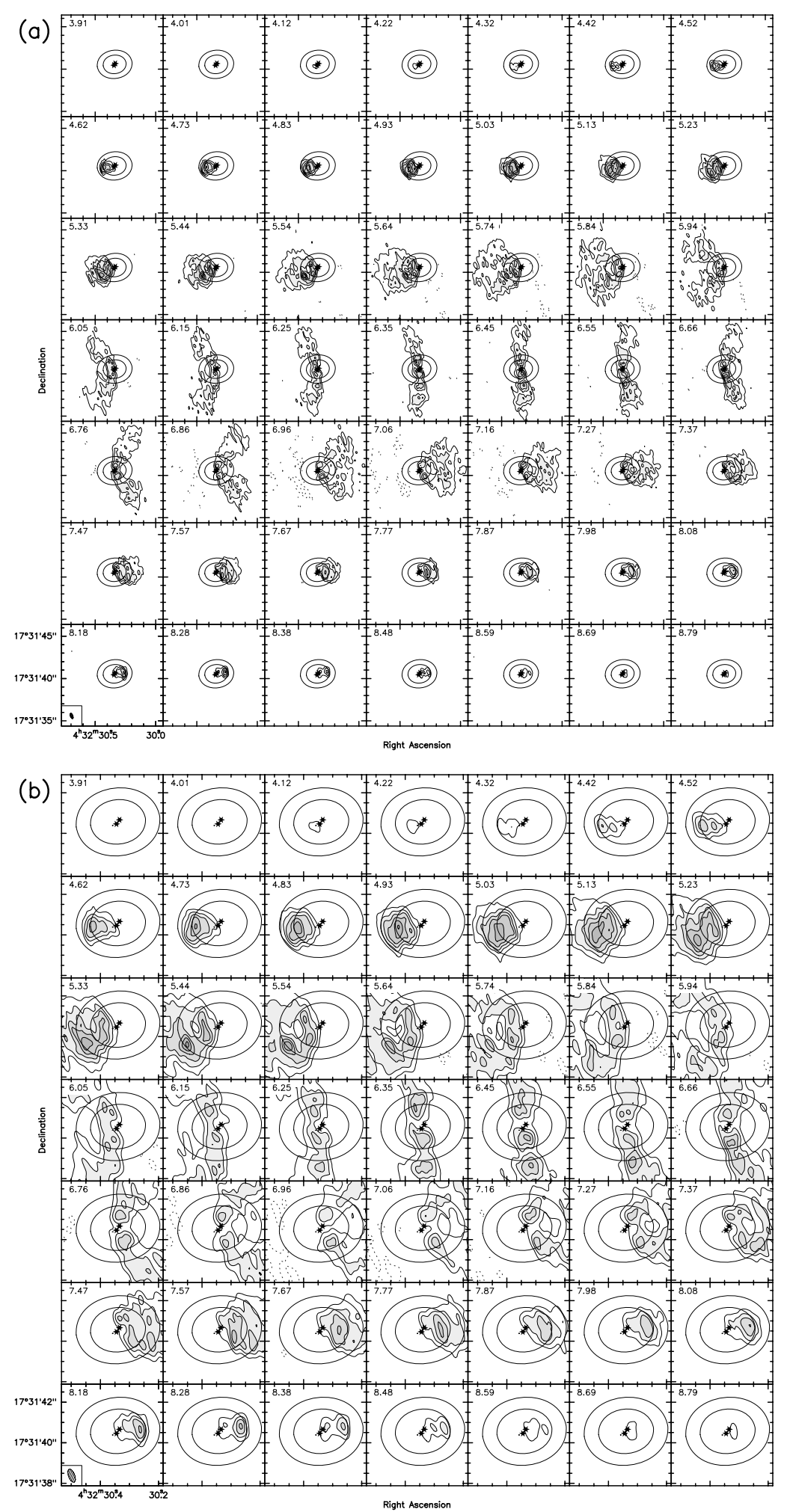


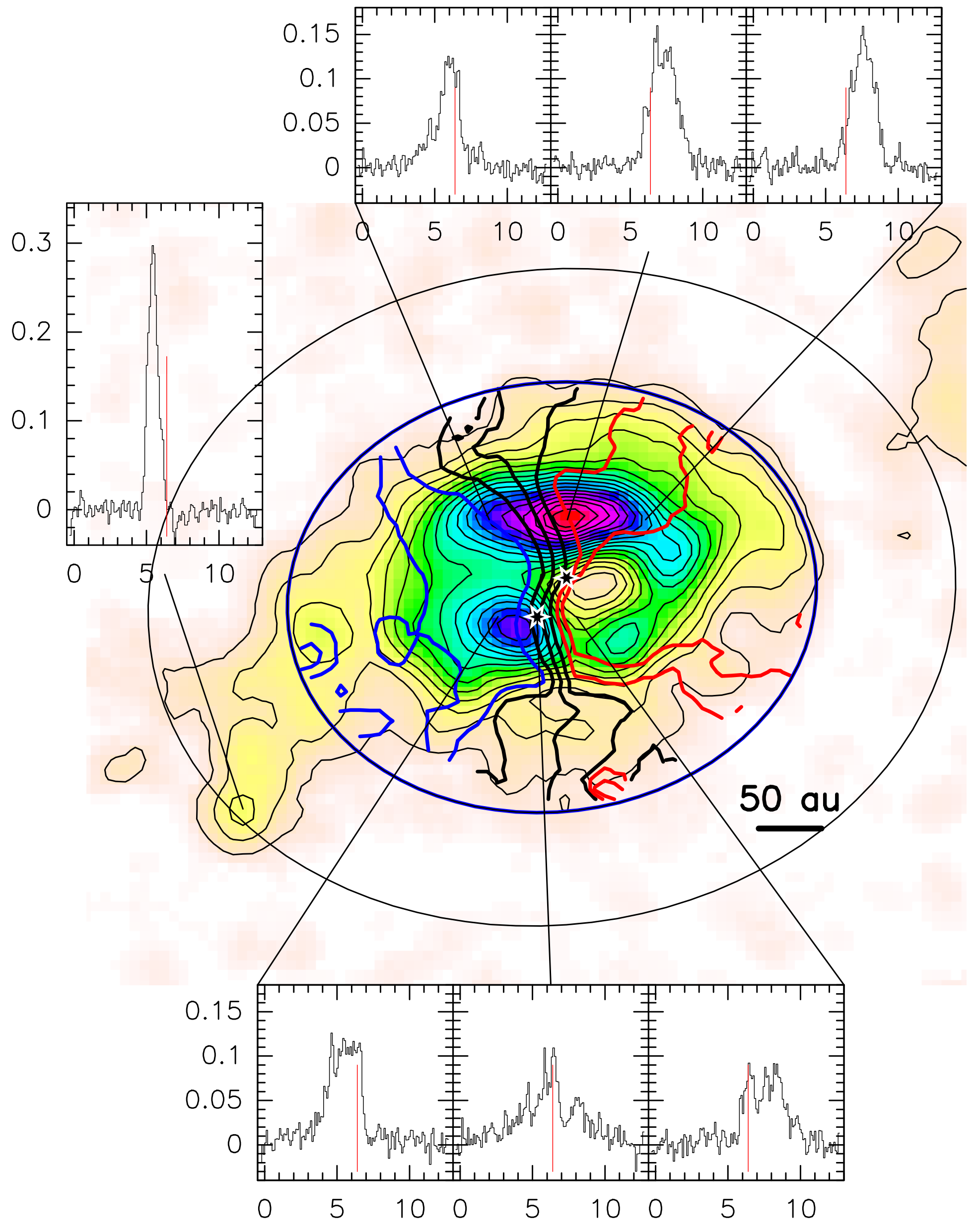




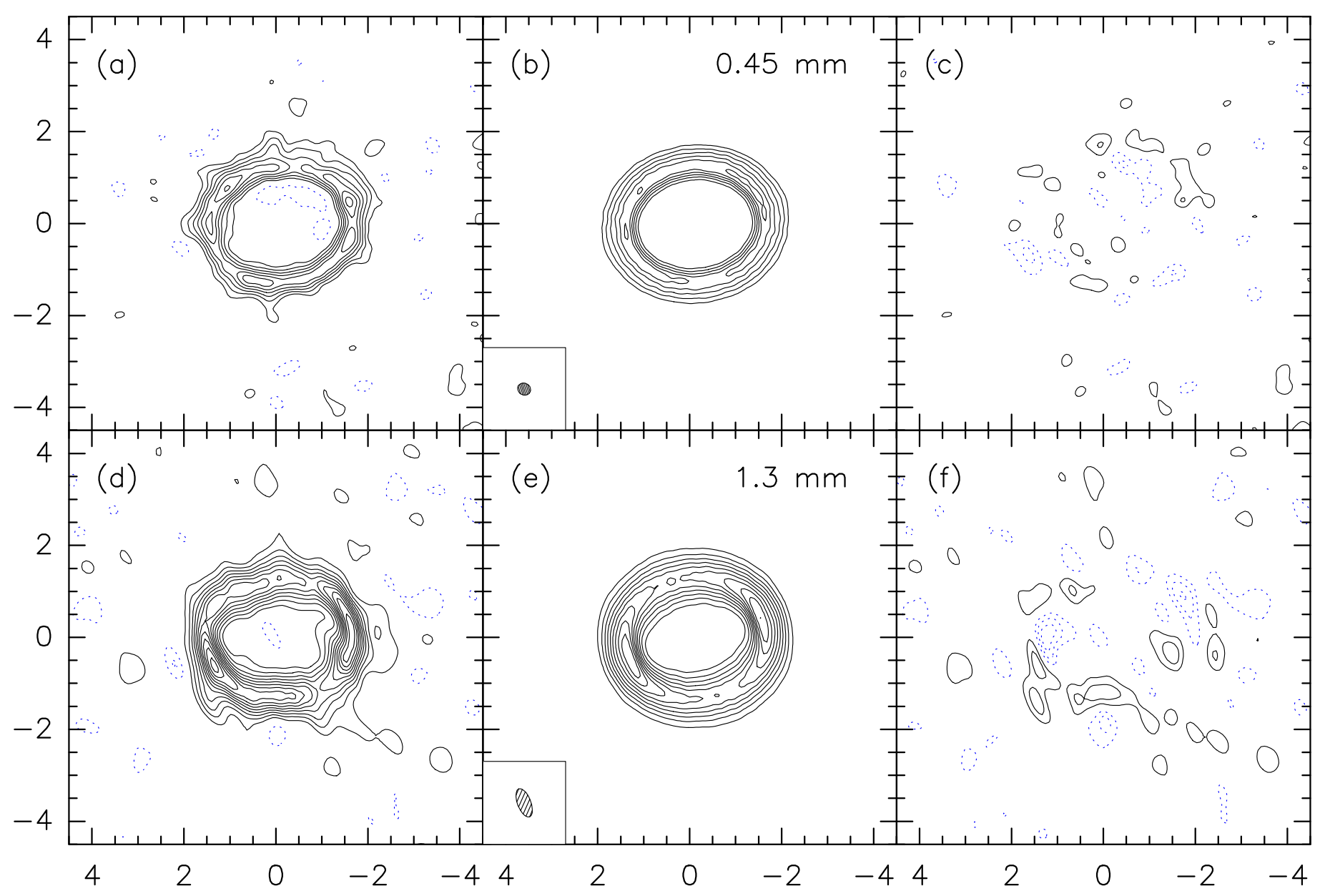

